\title{
The relative influence of different types of magnetotelluric data on joint inversions
}

\author{
Anna Gabàs and Alex Marcuello \\ GRC “Geodinàmica i Anàlisi de Conques", Departament de Geodinàmica i Geofisica, Universitat de Barcelona, \\ C/Martí i Franquès s/n, 08028 Barcelona, Spain
}

(Received February 20, 2002; Revised April 15, 2003; Accepted April 17, 2003)

\begin{abstract}
Diverse magnetotelluric data (apparent resistivity, phase or geomagnetic transfer function) used during the inversion process provide different information on the model as a consequence of data error. The relative influence of these data constitutes a subject of interest on the inversion process. This influence can be evaluated from the error ratio between two types of data; thus, when phase and the logarithm of apparent resistivity are involved, the wellknown ratio of one half is obtained. A new error ratio between the geomagnetic transfer function and the logarithm of apparent resistivity is presented. We deduced this ratio, which is bounded by one half of the amplitude of the geomagnetic transfer function. In order to verify this new ratio, we employed a technique based on the study of the RMS misfit, obtained after an intensive inversion computation whilst taking different error values for the different data. This technique was applied to synthetic and experimental data, and the results agree with the proposed value. This value should be taken into account for setting error floors when performing joint data inversion in order to obtain the same influence from the different data.
\end{abstract}

Key words: Magnetotelluric, inversion, geomagnetic transfer function.

\section{Introduction}

The magnetotelluric method is a geophysical technique based on the electromagnetic induction which determines the electrical conductivity distribution from the correlation between the components of the electromagnetic field. The magnetotelluric data (impedance tensor, $\boldsymbol{Z}$, and geomagnetic transfer function, $T$ ) are calculated from this electromagnetic field at every frequency. The impedance is determined as a linear relationship between horizontal components of electric $\left(\mathbf{E}_{\mathrm{h}}\right)$ and magnetic $\left(\mathbf{H}_{\mathrm{h}}\right)$ fields, and the geomagnetic transfer function as a linear relationship between vertical $\left(H_{z}\right)$ and horizontal components of the magnetic field:

$$
\begin{aligned}
& \mathbf{E}_{\mathrm{h}}=Z \cdot \mathbf{H}_{\mathrm{h}} \\
& H_{z}=T \cdot \mathbf{H}_{\mathrm{h}}
\end{aligned}
$$

2D inversion algorithms are currently used in magnetotelluric data interpretation, and the quality of the fit is measured by the RMS misfit between the data and the synthetic response of the model. These algorithms can handle different types of data (apparent resistivity, $\rho_{a}$, phase, $\varphi$, and geomagnetic transfer function, $T$ ) at the same time in order to reduce the non-uniqueness of the inverse problem. However, the incorporation of new data does not necessarily change the model obtained for two reasons: 1) the new data do not introduce additional information or constraints; 2) the inversion algorithm does not take into account new data given that the model is controlled by the old data. The latter reason raises the question of the relative influence (or weight) of the

Copy right(c) The Society of Geomagnetism and Earth, Planetary and Space Science (SGEPSS); The Seismological Society of Japan; The Volcanological Society of Japan; The Geodetic Society of Japan; The Japanese Society for Planetary Sciences. data on the inversion process. In these algorithms, the influence of an individual datum can be controlled by its error bar, which can be extended to all the data by using the error floor (or minimum possible error).

It is common knowledge that the ratio between the error of phase, $\varepsilon(\varphi)$, over the error of the logarithm of apparent resistivity, $\varepsilon\left(\ln \rho_{a}\right)$ is one half (e.g. Fischer and LeQuang, 1981). This ratio is directly obtained from the impedance, which is a complex magnitude.

The polar diagram of the impedance, $Z$, (Fig. 1) helps to clarify the meaning of the error, $\varepsilon(|Z|)$. From the definition of the apparent resistivity for a given frequency $f$ :

$$
Z=|Z| \cdot e^{i \varphi} \quad \text { and } \quad \rho_{a}=\frac{1}{2 \pi f \mu}|Z|^{2}
$$

and assuming that $\varepsilon(|Z|) \ll|Z|$, it can be easily obtained that

$$
\begin{gathered}
\varepsilon(\ln |Z|) \approx \varepsilon(\varphi) \approx \frac{1}{2} \varepsilon\left(\ln \left(\rho_{a}\right)\right) \\
\frac{\varepsilon(\varphi)}{\varepsilon\left(\ln \left(\rho_{a}\right)\right)}=\frac{1}{2}
\end{gathered}
$$

When using the inversion algorithms it is possible to select the suitable error floor for a given type of data. Result (5) means that when the same influence of the logarithm of apparent resistivity and phase on the inversion process is desired, the error floor of the $\varphi$ must be expressed as one half the one of $\ln \left(\rho_{a}\right)$. From this ratio a constant error floor is usually assumed for the whole range of frequencies. This assumption can be critical at low frequencies: The main component of error in the data has a statistical origin given that the other components of error can be neglected. 


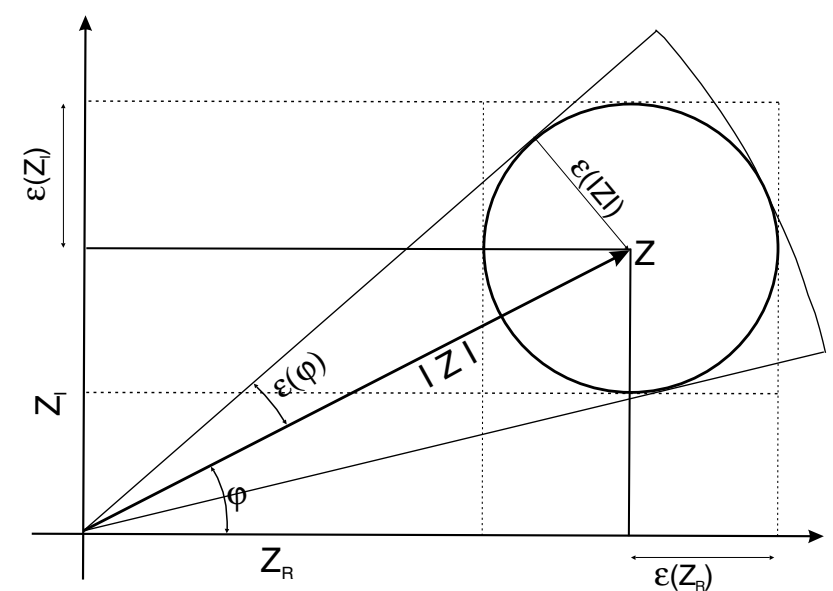

Fig. 1. Diagram of impedance, its phase, its real and imaginary parts, and their errors in the complex plane (modified from Bendat and Piersol, 1966).

When the frequency decreases at low frequencies, the value of $|Z|$ does not go up but the statistical error rises because of a diminution in the available data. Under these conditions, $\varepsilon(|Z|) /|Z|$ increases as frequency decreases, and the assumption of constant value for the error floor should be taken with caution. Nevertheless, in order to simplify the method presented in this paper, a constant error for all frequencies is assumed.

The aim of this paper is to obtain a similar relationship between the geomagnetic transfer function and the other magnetotelluric data in order to determine its relative influence. This will be useful when using the geomagnetic transfer function on the joint inversion process. If apparent resistivity is affected by static shift, the geomagnetic transfer function can give a more reliable model due to the magnetic field is less distorted (Ledo et al., 2002; Ogawa, 1999).

This study was carried out in two steps: first, we deduced the ratio between the errors of the geomagnetic transfer function and the impedance, which is based on Faraday's law; second, we devised a technique to verify the influence of the different data on the inversion algorithm using synthetic and experimental data (COPROD2); in particular, the ratio between the logarithm of apparent resistivity and the geomagnetic transfer function.

\section{Impedance-Geomagnetic Transfer Function Relationship}

We considered two-dimensional structures with the strike direction along the $x$-axis, and the $E$-polarization mode, which gives two magnetotelluric responses: impedance and geomagnetic transfer function.

The vertical component of the magnetic field is related to the horizontal component of the electric field according to Faraday's law:

$$
-i \omega \mu H_{z}=\frac{\partial E_{x}}{\partial y}
$$

Applying the linear relationships (1) and (2) to (6), we obtain

$$
T_{z y}=\frac{1}{-i \omega \mu} \frac{\partial \ln E_{x}}{\partial y} \cdot Z_{x y}=Y_{z x} \cdot Z_{x y}
$$

where $Y_{z x}$ is an admittance: $H_{z}=Y_{z x} E_{x}$. In order to simplify the notation and because there is no confusion, we avoid the use of subscripts in the responses $Z, T$ and $Y$.

Consequently, the error of the geomagnetic transfer function can be calculated using a simple error propagation scheme:

$$
\begin{aligned}
\varepsilon(|T|) & =\left|\frac{\partial T}{\partial Z}\right| \cdot \varepsilon(|Z|)+\left|\frac{\partial T}{\partial Y}\right| \cdot \varepsilon(|Y|) \\
& =|Y| \cdot \varepsilon(|Z|)+|Z| \cdot \varepsilon(|Y|) .
\end{aligned}
$$

And if the term with the unknown $\varepsilon(|Y|)$ is not taken into account, we obtain

$$
\varepsilon(|T|) \geq|Y| \cdot \varepsilon(|Z|)=\left|\frac{T}{Z}\right| \cdot \varepsilon(|Z|)
$$

which can be expressed by using (4) as

$$
\frac{\varepsilon(|T|)}{|T|} \geq \frac{\varepsilon(|Z|)}{|Z|} \approx \varepsilon(\ln |Z|) \approx \varepsilon(\varphi) \approx \frac{1}{2} \varepsilon\left(\ln \left(\rho_{a}\right)\right) .
$$

The previous expression allows us to state a ratio between the errors of $Z$ and $T$ for every frequency; and also, we can say (see Fig. 1) that $\varepsilon(|T|)=\varepsilon\left(T_{R}\right)=\varepsilon\left(T_{I}\right)$ because $T$ is a complex magnitude, and $T_{R}$ and $T_{I}$ are its real and imaginary parts. For simplicity only the error ratio $\varepsilon\left(T_{R}\right) / \varepsilon\left(\ln \left(\rho_{a}\right)\right)$ is studied, and the equation (9) is written as

$$
\frac{\varepsilon\left(T_{R}\right)}{\varepsilon\left(\ln \rho_{a}\right)} \geq \frac{|T|}{2} \text {. }
$$

This result can be extended to error ratios between other type of magnetotelluric data. An equivalent development could be used to deduce the error ratio $\varepsilon\left(T_{I}\right) / \varepsilon\left(\ln \left(\rho_{a}\right)\right)$.

The obtained ratio (10) depends on frequency and site (note that the phase-apparent resistivity ratio (5) does not depend on these). In order to avoid this dependency on the inversion process, we suggest taking the maximum value of $|T|$ to obtain a lower bound for this ratio:

$$
\frac{\varepsilon\left(T_{R}\right)}{\varepsilon\left(\ln \rho_{a}\right)} \geq \frac{\max |T|}{2} .
$$

This expression means that if we take an error of 0.1 for $\ln \left(\rho_{a}\right)$ (or $10 \%$ for $\rho_{a}$ ), it would be equivalent to an error of $0.05 \mathrm{rad}\left(\right.$ or $\left.2.8^{\circ}\right)$ in $\varphi$, and at least an error of $(0.05|T|)$ in $T$.

\section{Verification of Process and Results}

We devised a technique to study the influence of different data on the inversion algorithm in order to verify expression (11).

In accordance with the hypothesis that the influence of the data on the inversion algorithm is controlled by their error, this technique is based on the study of the RMS misfit obtained by multiple inversion processes.

In this procedure, the joint inversion is repeated by assigning given values of error to each type of magnetotelluric data. On inversion each type of data has the same error, which changes from inversion to inversion. This technique allows us to obtain a description of RMS as a function of error ratios. We assume that the suitable ratio is achieved when the 


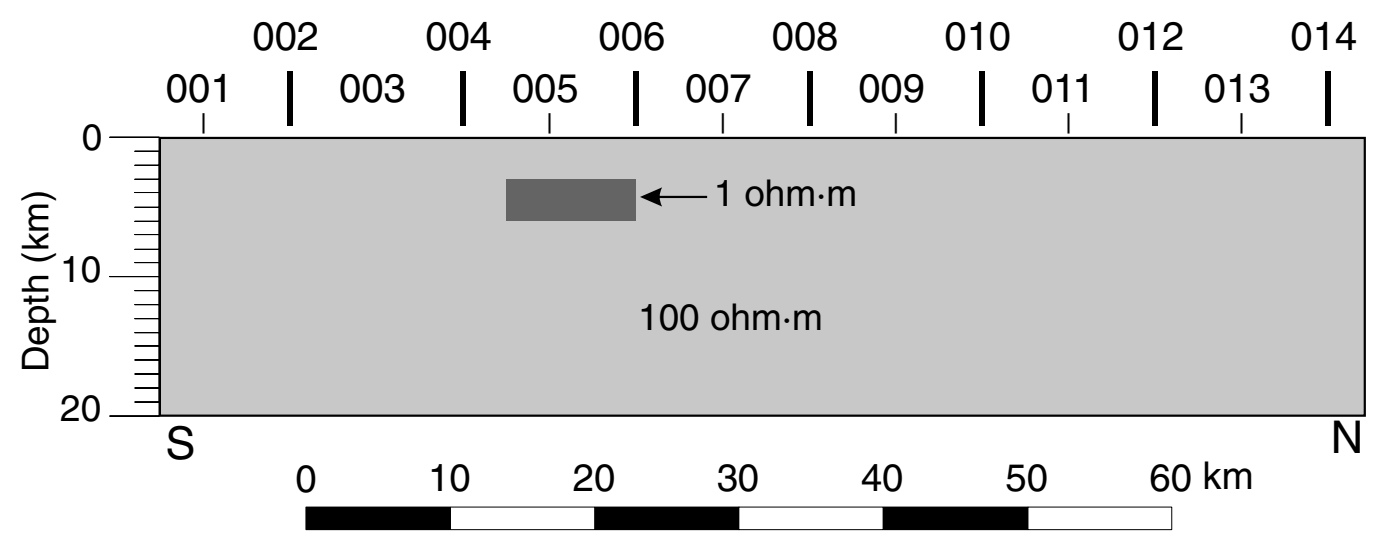

Fig. 2. Model used to generate synthetic data.

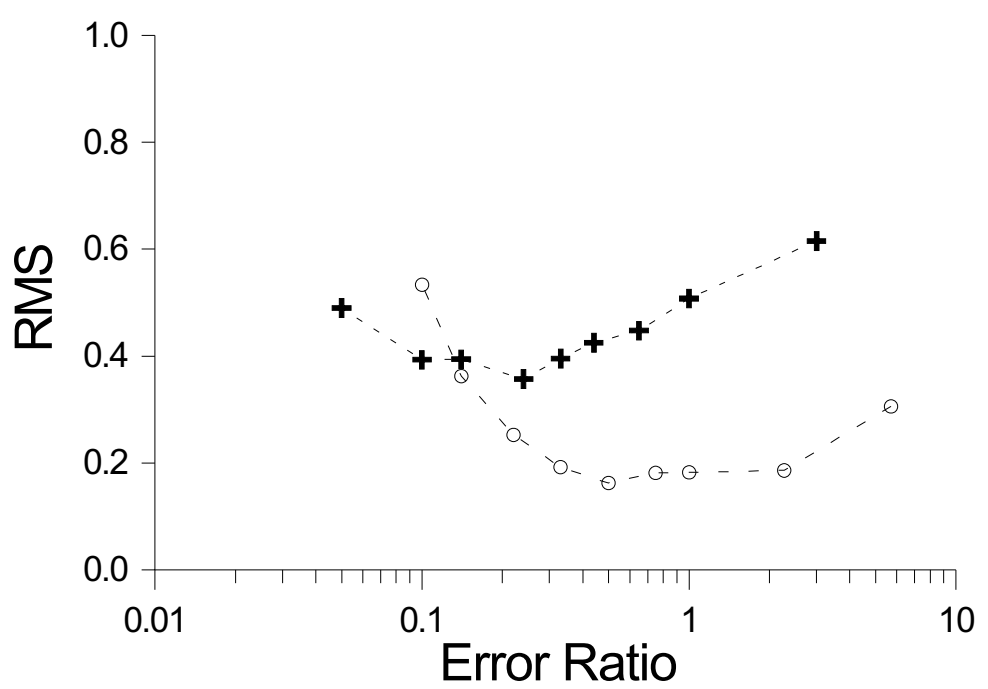

Fig. 3. For the synthetically generated data, behavior of the RMS versus: a) error ratio between phase and logarithm of apparent resistivity (circles); b) error ratio between geomagnetic transfer function (real part) and logarithm of apparent resistivity (crosses).

RMS obtained by the inversions reaches its minimum value. The RMS misfit is defined as:

$$
\mathrm{RMS}=\left(\frac{1}{N} \sum_{i=1}^{N} \frac{\left(d_{i}-f_{i}\right)^{2}}{\varepsilon_{i}^{2}}\right)^{1 / 2}
$$

where $d_{i}$ are the observed data, $f_{i}$ are the model responses, $\varepsilon_{i}$ are the observed data errors, and $N$ is the number of measured data.

The inversion process was performed using the REBOCC code of Siripunvaraporn and Egbert (2000), which enables us to take into account any type of data and any type of error on the inversion. The joint inversion was employed for two types of magnetotelluric data: a) $\varphi$ and $\ln \rho_{a}$ for calibration purposes since ratio (5) is well established; b) $T_{R}$ and $\ln \rho_{a}$, for verifying the proposed relationship (11).

Since the computation of RMS (12) depends on error values, $\varepsilon(\varphi)$ and $\varepsilon\left(\ln \rho_{a}\right)$ (case a), or $\varepsilon\left(T_{R}\right)$ and $\varepsilon\left(\ln \rho_{a}\right)$ (case b), they are changed without modifying their product to maintain a similar RMS level. With this constraint, the minimum RMS is always obtained at the same error ratio. For different error values the RMS will be different, but if the error ratio is the same, the models obtained from the inversion will not change.
The procedure was applied to synthetically generated data as well as to experimental data (COPROD2).

\subsection{Synthetic data}

Given that the quality of synthetic data is guaranteed, these data were used to verify the technique. To generate synthetic data, the model shown in Fig. 2 was considered. This consists of a host medium of $100 \Omega \cdot \mathrm{m}$, and a conductive structure of $1 \Omega \cdot \mathrm{m}$ with a rectangular section, $7.5 \mathrm{~km}$ width with the top at $3.1 \mathrm{~km}$, and the bottom at $6 \mathrm{~km}$. The distance between consecutive sites is $6 \mathrm{~km}$. The $E$-polarization data at 14 sites and 11 frequencies, ranging from $0.0008 \mathrm{~Hz}$ to 8 $\mathrm{Hz}$, were considered and were calculated with the algorithm of Wannamaker et al. (1986).

Nine inversions were performed with different values of error ratio, ranging from 0.05 to 5 on the logarithmic scale. The same initial model is used for every inversion, and an average of 20 iterations is carried out.

The results of these inversions are summarized in Table 1(a), Table 1(b) and Fig. 3, where circles are for the ratio $\left.\varepsilon(\varphi) / \varepsilon\left(\ln \rho_{a}\right)\right)$, and crosses, for the ratio $\varepsilon\left(T_{R}\right) / \varepsilon\left(\ln \rho_{a}\right)$. This figure represents the RMS misfit versus error ratios, where the horizontal axis is logarithmic. For this model, the highest value of $|T|$ is 0.46 , and analytical ratios (5) and (11) 

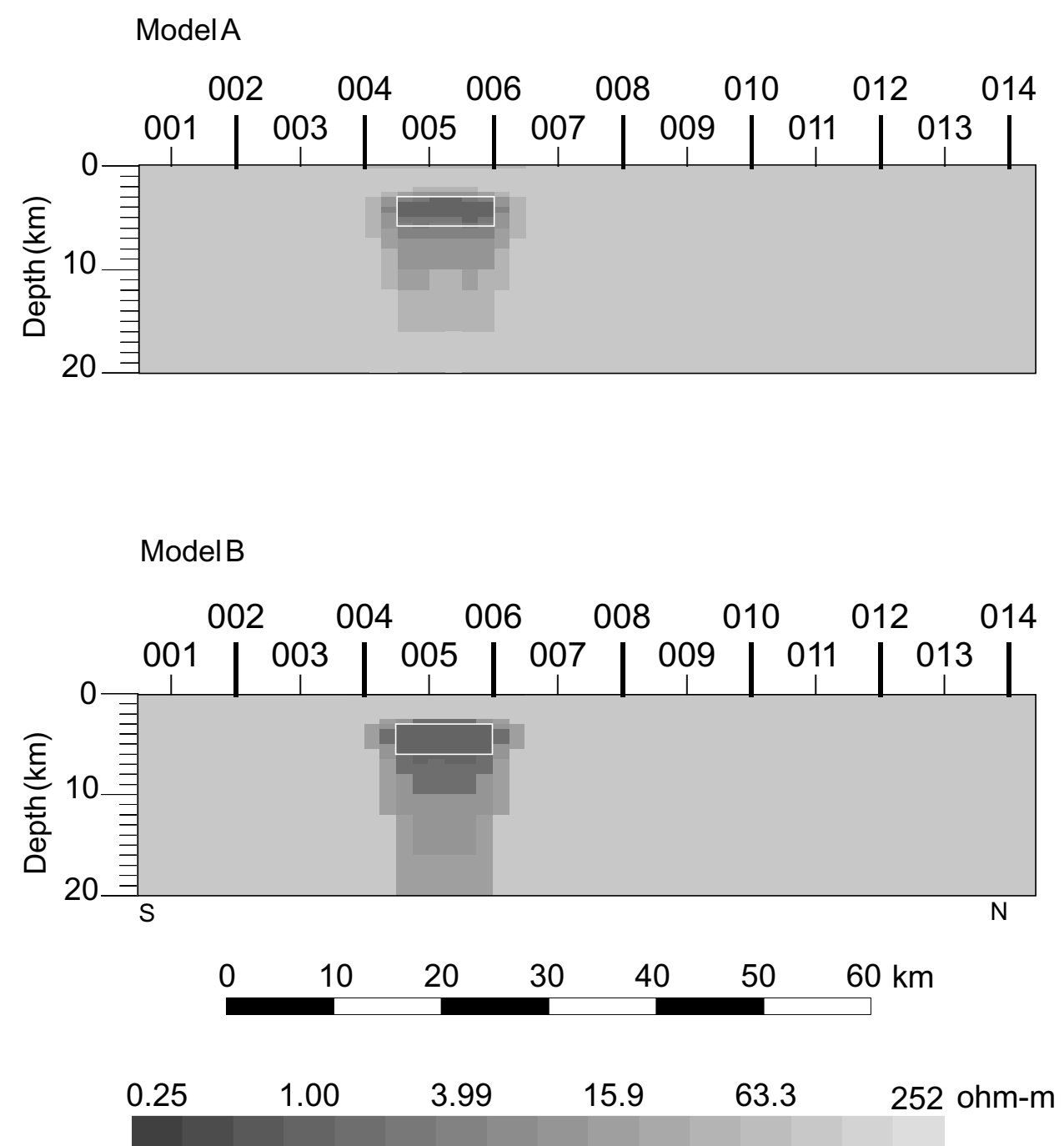

Fig. 4. Models obtained from the inversion of synthetic data $\left(T_{R}\right.$ and $\left.\ln \rho_{a}\right)$ for two different error ratios. Model A for $\varepsilon\left(T_{R}\right) /\left(\varepsilon \ln \rho_{a}\right)=0.24$ and model B for $\varepsilon\left(T_{R}\right) / \varepsilon\left(\ln \rho_{a}\right)=3$.

Table 1(a). Errors of logarithm of apparent resistivity and phase, and the corresponding error ratio for synthetic data.

\begin{tabular}{ccc}
\hline Error $(\ln ($ app.res $))$ & Error (phase) & Error ratio \\
\hline 0.007 & 0.04 & 5.71 \\
0.011 & 0.025 & 2.27 \\
0.015 & 0.015 & 1.00 \\
0.0186 & 0.014 & 0.75 \\
0.024 & 0.012 & 0.50 \\
0.03 & 0.01 & 0.33 \\
0.036 & 0.008 & 0.22 \\
0.043 & 0.006 & 0.14 \\
0.05 & 0.005 & 0.10 \\
\hline
\end{tabular}

give the values of 0.5 and 0.24 , which correspond to the minima of the RMS misfits observed in Fig. 3.

The models obtained by inverting $T_{R}$ and $\ln \left(\rho_{a}\right)$ are shown in Fig. 4. Even though both models recover the main features of the original model, the model A (error ratio of 0.24 ) is better than the model B (error ratio of 3.0).
Table 1(b). Errors of logarithm of apparent resistivity and real part of geomagnetic transfer function, and the corresponding error ratio for synthetic data.

\begin{tabular}{ccc}
\hline Error $(\ln ($ app.res. $))$ & Error $\left(T_{R}\right)$ & Error ratio \\
\hline 0.01 & 0.03 & 3.00 \\
0.015 & 0.015 & 1.00 \\
0.02 & 0.013 & 0.65 \\
0.025 & 0.011 & 0.44 \\
0.03 & 0.01 & 0.33 \\
0.038 & 0.009 & 0.24 \\
0.043 & 0.006 & 0.14 \\
0.05 & 0.005 & 0.10 \\
0.06 & 0.003 & 0.05 \\
\hline
\end{tabular}

\subsection{Experimental data}

The procedure above described is repeated with experimental data. We chose the well known COPROD2 data set (Jones, 1993) acquired in southern Saskatchewan and Manitoba (Canada); the $E$-polarization data at 27 sites and 19 


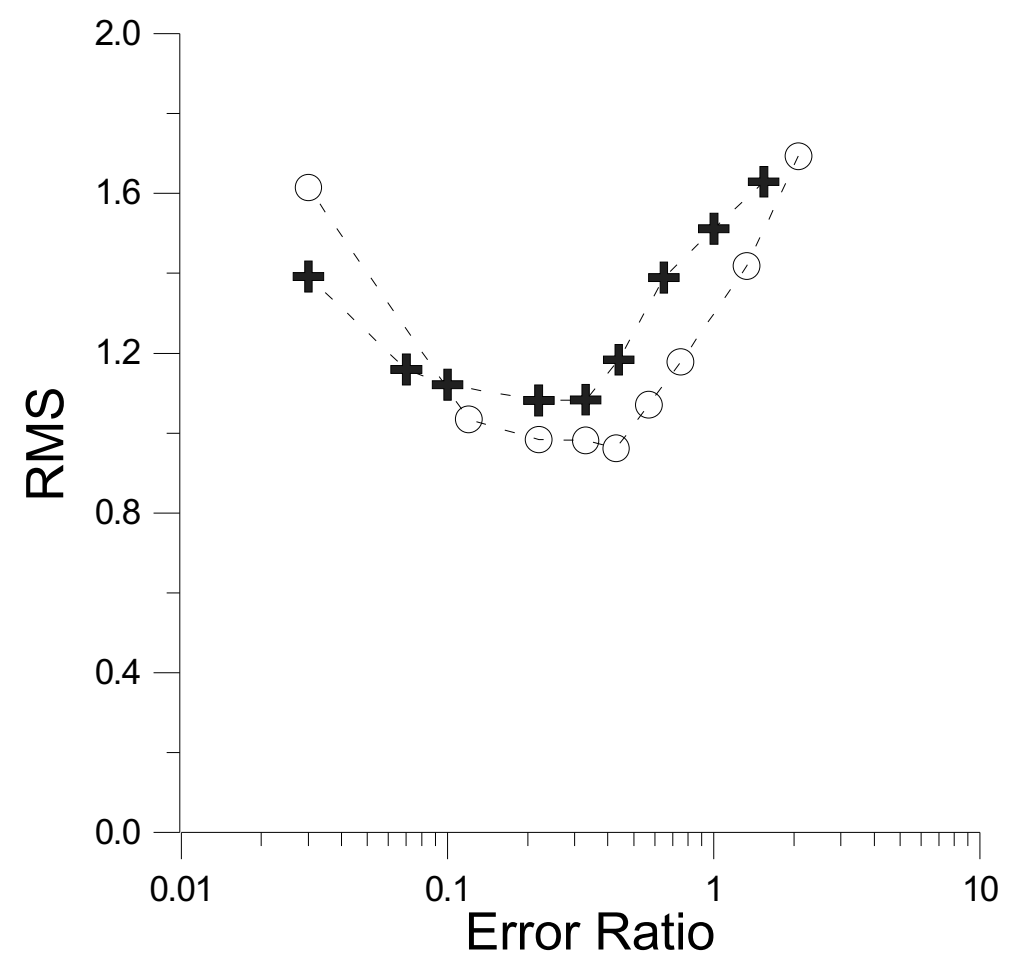

Fig. 5. For COPROD2 data set, behavior of the RMS versus: a) error ratio between phase and logarithm of apparent resistivity (circles); b) error ratio between geomagnetic transfer function (real part) and logarithm of apparent resistivity (crosses).

Table 2(a). Errors of logarithm of apparent resistivity and phase, and the corresponding error ratio for real data, COPROD2.

\begin{tabular}{ccc}
\hline Error (ln(app.res)) & Error (phase) & Error ratio \\
\hline 0.12 & 0.25 & 2.08 \\
0.15 & 0.20 & 1.33 \\
0.20 & 0.15 & 0.75 \\
0.23 & 0.13 & 0.57 \\
0.28 & 0.12 & 0.44 \\
0.30 & 0.10 & 0.33 \\
0.37 & 0.08 & 0.22 \\
0.50 & 0.06 & 0.12 \\
1.00 & 0.03 & 0.03 \\
\hline
\end{tabular}

frequencies, ranging from $0.0014 \mathrm{~Hz}$ to $1 \mathrm{~Hz}$, were considered. For these data, the $\max (|T|)$ is 0.75 , which suggests the value of 0.37 for the error ratio between the geomagnetic transfer function and the logarithm of apparent resistivity.

In line with the above criterion as in the case of the synthetic data, we also considered up to 11 different values of error ratio, ranging from 0.03 to 2 on the logarithmic scale. The same initial model is used for every inversion, and an average of 11 iterations is obtained.

The RMS misfit versus error ratio is represented in Fig. 5. The minima of the RMS misfits observed in this figure agreed with the given ratios (5) and (11). The chosen values of error are shown in Tables 2(a) and 2(b).

The models obtained for these minimum values are given in Fig. 6. The major conductors are similar in both cases,
Table 2(b). Errors of logarithm of apparent resistivity and real part of geomagnetic transfer function, and the corresponding error ratio for real data, COPROD2.

\begin{tabular}{ccc}
\hline Error (ln(app.res) $)$ & Error $\left(T_{R}\right)$ & Error ratio \\
\hline 0.13 & 0.20 & 1.54 \\
0.15 & 0.15 & 1.00 \\
0.20 & 0.13 & 0.65 \\
0.25 & 0.11 & 0.44 \\
0.30 & 0.10 & 0.33 \\
0.36 & 0.08 & 0.22 \\
0.50 & 0.05 & 0.10 \\
0.66 & 0.043 & 0.07 \\
1.00 & 0.03 & 0.03 \\
\hline
\end{tabular}

but the conductor under the site 503 is more outstanding in the model $\mathrm{B}$, because of the inclusion of the geomagnetic transfer functions.

\section{Discussion and Conclusions}

The error ratios between different types of magnetotelluric data account for their influence on inversion processes when different types of data are involved. In this paper we obtained an expression of the error ratio between the impedance and the geomagnetic transfer function.

The previous error ratio was verified with a technique based on the study of the RMS misfit after multiple inversion processes. In spite of the devised procedure had limitations associated with numerical computations, the results were satisfactory. 


\section{MODEL A}

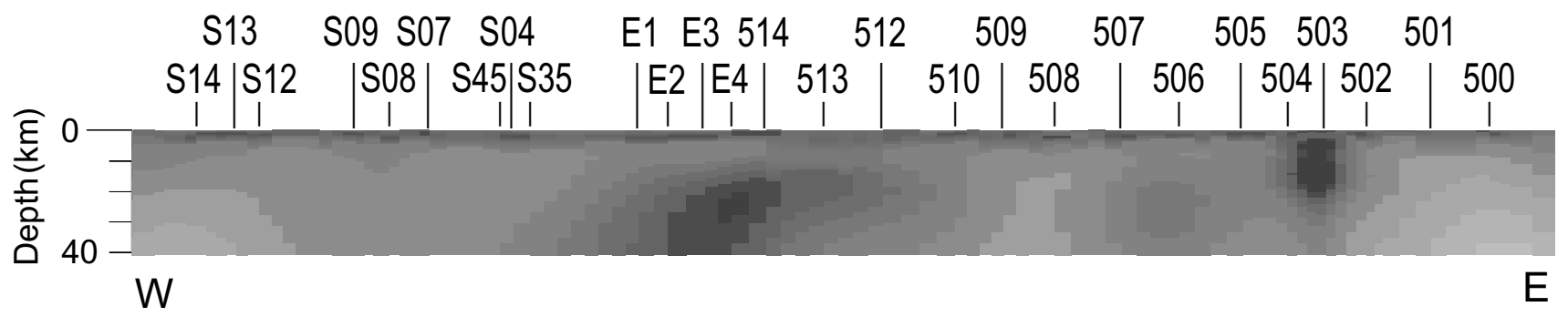

\section{MODEL B}

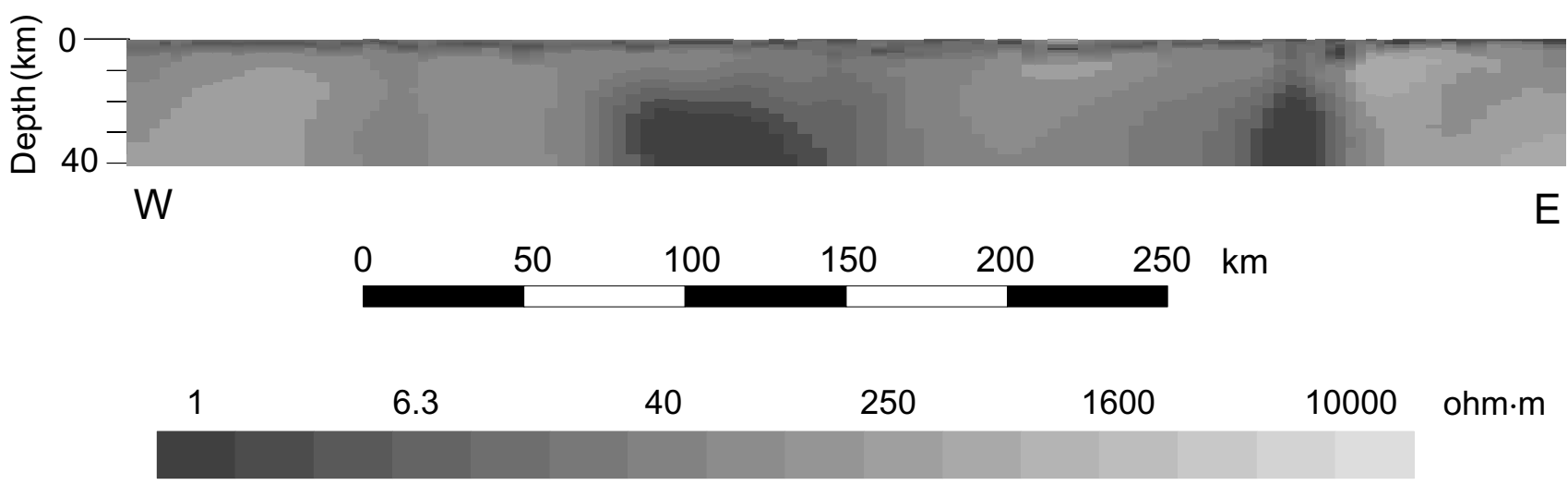

Fig. 6. Models obtained from the inversion of the COPROD2 data set corresponding with the minima RMS of Fig. 5: model A (for the ratio $\varepsilon(\varphi) / \varepsilon\left(\ln \left(\rho_{a}\right)\right)$, and model $\mathrm{B}$ (for the ratio $\varepsilon\left(T_{R}\right) / \varepsilon\left(\ln \rho_{a}\right)$ ).

The obtained ratio depends on the frequency and on the site because of the geomagnetic transfer function. For this reason we took the following expression as a lower bound:

$$
\varepsilon(|T|)=0.5 \quad \max |T| \varepsilon\left(\ln \rho_{a}\right) .
$$

In most fieldwork the geomagnetic transfer function $|T|$ is lower than one, and if this value is taken in the above expression, a value of one half is obtained. This case should be taken as a "physical" lower bound (e.g. this value was used by Ogawa et al., 1996), i.e., the maximum influence of the geomagnetic transfer function.

For field data, the errors of the geomagnetic transfer function and the impedance are obtained independently, and the ratio presented here shows the data with the most influence on the inversion process. In general, the impedance is more accurate, and the joint inversion of the apparent resistivity and the geomagnetic transfer function are biased by the former data.

The result will be useful when inverting the geomagnetic transfer function and the impedance elements at the same time for two reasons: a) it can indicate which data will exert the most influence on the inversion, and b) it will allow us to optimize the processes using the suitable data error on the inversion. The study about the error ratios opens a new perspectives for further works.

Acknowledgments. We should like to thank P. Queralt, J. Ledo and A. Jones for their helpful comments on this work, and W.
Siripunvaraporn and two anonymous referees for improving the manuscript. We should also like to acknowledge P. J. Savage of PanCanadian Petroleum Limited and A. Jones for the use of COPROD2 data set. This work was partially supported by the projects no. BTE2001-3650 and REN2002-4538-C02-01 of Spanish Ministry of Science and Technology.

\section{References}

Bendat, J. and A. Piersol, Measurement and Analysis of Random Data, New York, Wiley, 1966

Fischer, G. and B. V. LeQuang, Topography and minimization of the standard deviation in one-dimensional magnetotelluric modeling, Geophys. J. R. astr. Soc., 67, 279-292, 1981.

Jones, A. G., The COPROD2 dataset: Tectonic setting, recorded MT data, and comparison of models, J. Geomag. Geoelectr., 45, 933-955, 1993.

Ledo, J., A. Gabas, and A. Marcuello, Static shift levelling using geomagnetic transfer functions, Earth Planets Space, 54, 493-498, 2002.

Ogawa, Y., Constrained inversion of COPROD-2S2 dataset using model roughness and static shift norm, Earth Planets Space, 51, 1145-1151, 1999.

Ogawa, Y., A. G. Jones, M. J. Unsworth, J. R. Booker, X. Lu, J. A. Craven, J. Permelee, and C. Farquharson, Deep electrical conductivity structures of the Appalachian Orogen in the southeastern U.S., Geophys. Res. Lett., 23, 1597-1600, 1996.

Siripunvaraporn, W. and G. Egbert, An efficient data subspace inversion method for 2-D magnetotelluric data, Geophysics, 65, 791-803, 2000.

Wannamaker, P. E., J. A. Stodt, and L. Rijo, A stable finite element solution for two-dimensional magnetotelluric modeling, Geophys. J. R. astr. Soc., 88, 277-296, 1986.

A. Gabàs (e-mail: agabas@geo.ub.es) and A. Marcuello (e-mail: alex.marcuello@ub.edu) 